\title{
Knowledge and attitude of dentists, dental auxiliaries, and students regarding the COVID-19 pandemic in Saudi Arabia: A Cross- Sectional Survey
}

\author{
Suliman Yousif Shahin \\ Imam Abdulrahman Bin Faisal University \\ Amr Said Bugshan ( $\sim$ abugshan@iau.edu.sa ) \\ Imam Abdulrahman Bin Faisal University \\ Khalid Salman Almulhim \\ Imam Abdulrahman Bin Faisal University \\ Mishali Saud AlSharief \\ Imam Abdulrahman Bin Faisal University \\ Yousif Al Dulaijan \\ Imam Abdulrahman Bin Faisal University \\ Faisal Dhaifallah Alqarni \\ Imam Abdulrahman Bin Faisal University
}

\section{Research Article}

Keywords: Awareness, Dentists, Dental Student, COVID-19, SARS-CoV-2

Posted Date: August 12th, 2020

DOI: https://doi.org/10.21203/rs.3.rs-57059/v1

License: @ (1) This work is licensed under a Creative Commons Attribution 4.0 International License. Read Full License 


\section{Abstract}

Background: This study aimed to assess the knowledge and attitude of dental professionals in Saudi Arabia regarding severe acute respiratory syndrome coronavirus 2 (SARS-CoV-2) and coronavirus disease 2019 (COVID-19).

Methods: A questionnaire was developed to assess various dental professionals from both governmental and private sectors through online and social media outlets.

Results: A total of 1,033 responses were collected. Moreover, $63.4 \%$ of respondents (63.4\%) were working in hospitals. Of all respondents, $44.9 \%, 33.4 \%$, and $21.7 \%$ were in governmental clinics, academia, and private sector, respectively. Overall knowledge on incubation period and route of transmission of SARS-CoV-2 was satisfactory across all dental professions. All dental professionals except for dental assistants demonstrated adequate knowledge on the recommended hand-soap cleaning time $(p<0.001)$. Most dental professionals displayed unsatisfactory knowledge and disagreement on the survival of SARS-CoV-2 outside the host $(p<0.001)$. Furthermore, $75.1 \%$ of respondents were reluctant to treat a suspected COVID-19 patient, and $92 \%$ participants believed that the mode of transmission was droplet inhalation. Fever, coughing, and shortness of breath were identified as the most common symptoms of COVID-19. Most standard methods of prevention in the dental office were selected by at least $50 \%$ of participants.

Conclusions: An appropriate level of knowledge on SARS-CoV-2 incubation period and signs and symptoms of COVID-19 was noted among all dental professionals in Saudi Arabia. Perceived survival outside the host was modest. A certain level of apprehension toward suspected SARS-CoV-2 patients existed among most professionals. Pandemic-awareness campaigns are essential among healthcare providers.

\section{Background}

On January 30, 2020, the World Health Organization (WHO) ${ }^{1}$ announced a public health emergency of international significance over a global pneumonia epidemic. The WHO called the novel viral pneumonia "coronavirus disease 2019 (COVID-19)" on February 11, 2020. The first few cases of COVID-19 were identified in December 2019 in Wuhan, China, after genome sequencing of samples from patients presenting with pneumonia symptoms of unknown etiology. ${ }^{1}$ The virus then spread to other provinces of China within weeks and became a global pandemic within a few months. ${ }^{2}$ On March 2, 2020, Saudi Arabia announced its first case of severe acute respiratory syndrome coronavirus 2 (SARS-CoV-2) infection. Since then, the Saudi Ministry of Health has urged the general population to engage in social distancing and adopt sanitary habits and self-quarantine for a period of 14 days if the individual has recently been residing out of the kingdom. Coronaviruses are enveloped positive-stranded RNA viruses with a crown-like appearance due to the presence of glycoprotein projections on their surfaces. ${ }^{3}$ The virus strain is SARS-CoV-2, which shares the same host receptor with SARS-CoV, namely, the human angiotensin-converting enzyme 2. The natural host of SARS-CoV-2 may be the bat as it showed $96.2 \%$ wholegenome identity to BatCoV RaTG13. ${ }^{4}$

The common clinical symptoms of patients with novel viral pneumonia include fever, cough, myalgia, and exhaustion with an irregular chest computed tomography scan, and the less common symptoms are sputum development, headache, hemoptysis, and diarrhea. ${ }^{5,6}$ The typical transmission routes of novel coronaviruses include direct (transmission through coughing, sneezing, and droplet inhalation) and contact (contact with oral, nasal, and eye mucous membranes). ${ }^{7}$

The dental professional team is considered a high-risk group. Pathogenic microorganisms may be transmitted in dental settings through inhalation of airborne microorganisms that can stay trapped in the air for long periods; ${ }^{8}$ direct contact with blood, oral fluids, or other patient materials; ${ }^{9}$ contact of the conjunctival, nasal, or oral mucosa with droplets and microorganism-containing aerosols produced from an infected person and propelled by coughing and talking at a short distance without a mask ${ }^{10,11}$ or using high-pressure irrigation systems such as the handpiece or ultrasonic scalers; and indirect contact with contaminated instruments and/or environmental surfaces. ${ }^{12}$ Therefore, it is crucial for the dental professional team to practice preventive measures against SARS-CoV-2 infection by focusing on hand hygiene, adequate hand-soap cleaning time of approximately 40 seconds, ${ }^{13}$ and personal protective equipment (PPE) in an adequately ventilated space and exercise caution when performing aerosol-generating procedures. 
The aim of this study was to assess the knowledge, attitude, and educational needs of dentists and dental auxiliaries and students in Saudi Arabia to SARS-CoV-2 as they are considered a high-risk group for infection and investigate the potential gaps in knowledge that may affect health and safety in the dental workplace.

\section{Methods}

A cross-sectional survey was conducted after ethical approval (EA\# 202049) was obtained from the Scientific Research Unit at the College of Dentistry, Imam Abdulrahman Bin Faisal University, Dammam, Saudi Arabia. The questionnaire was developed and further reviewed for face validation by two independent local reviewers. Major governmental hospitals, private clinics, and academic universities in various cities of all the provinces of Saudi Arabia were approached. A corresponding link was sent to all targeted samples using different methods, including emails, text messages, and social media outlets. The targeted sample included dental students, dental interns, dental hygienists, dental assistants, general dentists, dental specialists, and consultants working in hospital and nonhospital governmental and private sectors. To ensure the clarity and validity of the questions, a pilot test of the questionnaire was initially performed on a group of 100 dental professionals.

The construct of the survey was based on the latest literature resources about the new SARS-CoV-2 and COVID-19, including its characteristics, signs and symptoms, route of transmission, and prevention methods. The first part of the questionnaire included the demographic information of the participants, that is, their age group, sex, geographic location of practice within Saudi Arabia, type of profession, primary work sector (academia, government, or private), and work setting (hospital or nonhospital). The second part of the questionnaire assessed the participants' awareness of the basic knowledge of the new SARS-CoV-2 and COVID-19. Dental professionals were asked to provide their opinions about COVID-19 signs and symptoms, route of viral transmission, incubation period, and viability outside the human body. The last part of the questionnaire included the methods of prevention and their attitude toward treating SARS-CoV-2-infected patients.

SPSS-20.0 was used for statistical data analysis (IBM Corp. Released 2011. IBM SPSS Statistics for Macintosh, Version 20.0. Armonk, NY, USA: IBM Corp.). The results of all categorical variables are presented in terms of frequencies and percentages. The chi-square test was performed to compare the factors between work sectors, work settings, and professionals. All tests were performed at a significance level of $a=0.05$.

\section{Results}

Out of 1,033 responses, 20 were omitted as they were from outside Saudi Arabia. Some of the responses were missing due to incomplete reporting by some of the participants.

\subsection{Reliability and validity}

A pilot sample size of 100 participants was selected for the reliability and validity of the research instrument. After standardizing all items, reasonably good interrater reliability was found (Cronbach's alpha $=0.788$ ). Questionnaire face validation was performed by two independent local experts.

\subsection{Demographic results}

More than half of the participants were female, accounting for $53.5 \%(n=542)$ of the study sample, while men accounted for $46.5 \%$ $(n=471)$ of the study sample. Half of the participants were in the age group of 20 to $<30$ years of age $(50 \%)$. Followed by 301 participants in the age group of 30 to $<40$ years $(29.7 \%), 134$ were in the age group of 40 to $<50$ years $(13.2 \%)$, and 71 were in the age group of $\geq 50$ years of age (7\%). Among the professions, the highest number of responders were specialists/consultants $(n=271$, $26.8 \%)$, followed by dental students $(n=197,19.5 \%)$, general dentists $(n=181,17.9 \%)$, dental assistants $(n=156,15.4 \%)$, postgraduate residents $(n=92,9.1 \%)$, dental interns $(n=76,7.5 \%)$, and dental hygienists $(n=37,3.7 \%)$.

\subsection{Awareness of SARS-CoV-2 and bivariate analysis results}

When evaluating the awareness of the survival period of SARS-CoV-2 outside a host, approximately half of the responders $(n=496$, $49.2 \%)$ believed that the survivability of the virus outside the host was a couple of hours. The remaining responders were as follows: $360(35.7 \%)$ believed that the survivability of the virus was a couple of days, 71 (7\%) believed it was a couple of weeks, and 82 (8.1\%) did not know. In the between-group comparison, the survivability of SARS-CoV-2 outside a host among different work settings and 
sectors was not significantly different $(p>0.05)$. However, when evaluating the various dental professions, a statistically significant difference was found among the various professions $(p<0.001)$. Among dental assistants, $17.9 \%(n=28)$ believed that the survivability of SARS-CoV-2 was a couple of weeks, whereas only between $2.8 \%$ and $7.6 \%$ of the rest of the dental professionals believed the same concept. Furthermore, $48.3 \%(n=131)$ of specialists and consultants believed that the survivability of SARS-CoV-2 was a couple of days, while only $23.7 \%(n=18), 25.6 \%(n=40)$, and $26.4 \%(n=52)$ of the interns, assistants, and students believed the same concept. This indicates a statistically significant disagreement among dental professionals on the survivability of SARS-CoV-2 outside the host.

The awareness of the recommended hand-soap cleaning time to prevent SARS-CoV-2 infection was distributed as follows: 561 (55.6\%) responded as 40 s; 277 (27.4\%), 20 s; 155 (15.3\%), 60 s; and 17 (1.7\%), not knowing the recommended hand-soap cleaning time for prevention. Again, in the between-group comparison, the recommended hand-soap cleaning time to prevent SARS-CoV-2 infection among different work settings and sectors was not significantly different $(p>0.05)$. However, when evaluating the various dental professions, a statistically significant difference was found among the various professions with regard to the recommended hand-soap cleaning time $(p<0.001)$. The majority of dental professionals, except for dental assistants, responded in the range of $55.9 \%-69.4 \%$ on a recommended time of $40 \mathrm{~s}$, whereas only $25 \%(n=39)$ of dental assistants answered the recommended $40 \mathrm{~s}$ of hand-soap cleaning time to prevent SARS-CoV-2 contamination.

Regarding the questions about willingness to treat a suspected COVID-19 patient, approximately three-quarters of dental professionals $(n=756,75.1 \%)$ did not want to treat a suspected COVID-19 patient. Bivariate analyses for between-group comparisons among the various groups of work settings and sectors and dental professions for this question were not statistically different $(p>0.05)$. Tables 2 and 3 illustrate the knowledge and awareness of SARS-CoV-2 and COVID-19 by work settings and sectors and dental professions. The Pearson chi-square results are shown as well.

The majority of the 1,013 participants $(n=932,92 \%)$ believed that the route of transmission was through droplet spread (sneezing or coughing). Approximately half of the participants ( $n=548$ dental professionals, $54.1 \%$ ) believed that the virus could be transmitted through direct contact with saliva, blood, or other body fluids. Moreover, only approximately a third of the respondents believed that transmission could occur via direct skin-to-skin contact and airborne transmission with $312(30.8 \%)$ and $305(30.1 \%)$ responses, respectively.

Almost all of the respondents believed fever to be a sign and symptom of COVID-19 with 1,000 responses (98.7\%). Furthermore, 944 (93.2\%) respondents believed coughing to be among the signs and symptoms. In addition, $940(92.8 \%)$ respondents believed shortness of breath to be among the common signs and symptoms of the disease. The response for the remaining COVID-19 signs and symptoms were as follows: 736 (72.7\%) responses for headache; 671 (66.2\%), shortness of breath; 492 (48.6\%), muscle pain; 323 (31.9\%), diarrhea; 295 (29.1\%), nasal congestion; 187 (18.5\%), vomiting; and 32 (3.2\%), skin rash.

The methods of SARS-CoV-2 transmission prevention in dental clinics among participants were believed to be as follows (from the highest response to the lowest): PPE (gloves, masks, and wrapping) with 973 (96.1\%) responses; hand-soap cleaning, 960 (94.8\%); clinic surface disinfection, 877 (86.6\%); hand sanitizers, 873 (86.2\%); rubber dam isolation, 620 (61.2\%); utilization of an isolated clinic, 592 (58.4\%); adequate ventilation, 579 (57.2\%); use of preoperative chlorhexidine mouthwash, 360 (35.5\%); and use of preoperative hydrogen peroxide mouthwash, 207 (20.4\%). Table 4 summarizes the responses of all dental professionals with regard to the awareness of the route of SARS-CoV-2 transmission, signs and symptoms of COVID-19, and methods of transmission prevention in dental clinics.

\section{Discussion}

Due to its high mortality rate, SARS-CoV-2 and the associated debilitating COVID-19 are an emerging topic that has recently received great attention and underwent intense investigation. The pandemic is highly infectious as the number of infected patients exceeds 2 million worldwide in $<6$ months, as reported by the WHO. ${ }^{14}$

This survey assessed the degree of awareness of dental professionals as they are typically in close contact with patients when delivering oral healthcare services.

Based on our results, the overall knowledge about the means of transmission, associated symptoms, and prevention of SARS-CoV-2 was satisfactory. The overall knowledge about the incubation period, route of transmission, and recommended hand-soap cleaning

Page $4 / 11$ 
time to prevent SARS-CoV-2 was adequate across all dental professions, except for dental assistants, where only $25 \%$ of them believed the recommended hand-soap cleaning time was 40 seconds. This clearly emphasizes the significance of the adequacy of viral prevention knowledge across all dental professionals and auxiliaries during pandemic periods. Overall, these results show an improvement in knowledge compared with the results found in a previous survey of dentists in Saudi Arabia about SARS-CoV. ${ }^{15}$ Although modestly answered among all professionals, the highest response was among dental specialists/consultants, with only $48.3 \%$ believing that the survivability of SARS-CoV-2 outside the host was a couple of days. There seemed to be a significant disagreement among the dental professionals regarding the question of SARS-CoV-2 survivability $(p<0.001)$. Regarding general knowledge about SARS-CoV-2, our study showed a relatively lower percentage of individuals who answered "I do not know" in questions regarding the incubation period and recommended hand-soap cleaning time $(0.69 \%$ and $1.69 \%$, respectively) compared with virus survival outside the host (10.04\%). This may be due to the fact that local health authorities have utilized all media outlets toward a community-wide awareness campaign covering the pandemic, which had hand-soap cleaning time being one of the most shared preventive methods promoted. In contrast, the survival of the virus outside the host may be confusing due to various survival times related to different surfaces. ${ }^{8,16}$ This further emphasizes the significance of knowledge on virus survival to ensure adequate preventive measures in the working environment while treating suspected COVID-19 patients.

The majority of respondents, regardless of professional category, were reluctant to treat a patient under suspicion of having COVID-19, which indicated a certain level of apprehension. This is thought to be due to the general guidelines conveyed to caregivers by local authorities, that is, to limit dental procedures to urgent or emergency cases only and refrain from elective procedures. When it came to willingness to treat suspected patients with COVID-19, dental hygienists (38.89\%) were most willing to treat suspected patients. In contrast, postgraduate dental residents (17.39\%) were least willing compared with the remaining groups. This is an interesting outcome as dental hygiene is considered to have mostly elective procedures compared with general or specialized dentistry. Future studies addressing the level of apprehension among dental professionals are warranted.

Regarding the question about modes of transmission, the majority of the participants (92\%) believed that the mode of transmission was droplet infection, followed by direct fluid transmission (54.1\%) and direct skin-to-skin contact (30.8\%). However, 30.1\% of the participants also believed that the virus was airborne transmittable, which is clearly a misconception, as the disease is transmitted by aerosols. ${ }^{3}$ The present study showed that the majority of the participants identified fever, coughing, and shortness of breath as the most common symptoms related to COVID-19 (98.7\%, 93.2\%, and 92.8\%, respectively), which indicated a high degree of knowledge pertinent to this area. This is an intuitive finding considering that fever screenings have been increasingly prevalent at establishment entrances throughout the pandemic. Furthermore, previous epidemics of SARS-CoV and Middle East respiratory syndrome-related coronavirus (MERS-CoV) may explain the degree of knowledge regarding common respiratory symptoms of coronaviruses.

To prevent SARS-CoV-2 infection in dental clinics, dental professionals should follow standard precautions, which include appropriate hand hygiene, personal protective barriers (gloves, masks, face shields), use of preoperative mouthwash (1\% hydrogen peroxide), rubber dam isolation, and implementation of effective and strict disinfection measures. ${ }^{17}$ It is worth highlighting that $54.6 \%$ and $51.6 \%$ of dental assistants and hygienists, respectively, believed that the use of preoperative chlorhexidine mouthwash (0.12\%), which is antibacterial, was a preventative measure against SARS-CoV-2. On the other hand, $84.9 \%$ and $93.5 \%$ of dental assistants and hygienists did not believe that rubber dam isolation was a significant measure of SARS-CoV-2 prevention. This implies the presence of a gap in knowledge regarding preventive measures among the dental team.

The representative sample of 1,013 dental professionals covering all regions of Saudi Arabia is a strength of this study as it is consistent with the distribution of the population within each region. ${ }^{18}$ Furthermore, a thorough distribution among all the dental professionals and dental work settings was achieved to act as a realistic representation of the country's dental work force. To the best of our knowledge, this is the first representative survey to provide insight on the knowledge and perception of dentists and dental auxiliaries and students on SARS-CoV-2 in Saudi Arabia. Furthermore, the distribution of male and female participants was balanced (46.5\% and $53.5 \%$, respectively). However, this study has several limitations. In addition to the general limitations of a cross-sectional study and online surveys, one of our limitations was the dichotomous nature of the question as to whether the practitioner was willing to treat a SARS-CoV-2 patient. Information pertaining participants who answered "No" but who would otherwise be willing to treat emergency patients only may have been lost as the options were only "Yes" or "No," especially with a considerable number of dental procedures being elective or at least of a nonurgent nature. In addition, with the high volume of information influx from media outlets in combination with a higher than average viewership due to curfews and lockdowns, the status quo of general perceptions may shift on 
a weekly or even daily basis, rendering this survey limited to a specific snapshot of time. This study can be used as a benchmark for general knowledge and attitude toward pandemics in developing countries with an established dental healthcare system.

\section{Conclusion}

An adequate level of knowledge about the SARS-CoV-2 incubation period and signs and symptoms of COVID-19 among all dental professionals in Saudi Arabia was demonstrated. The knowledge of the required hand-soap cleaning time for the prevention of SARSCoV-2 among all dental professions was satisfactory, except for dental assistants. However, knowledge about the survival of the virus outside the host was modest. Furthermore, a certain level of apprehension toward suspected COVID-19 patients existed among all dental professionals. During pandemics, an effort to increase knowledge among dentists and dental auxiliaries needs to be reinforced within the dental professional team.

\section{Declarations}

\section{- Ethics approval and consent to participate}

The Scientific Research Unit at the College of Dentistry, Imam Abdulrahman Bin Faisal University approved this study. A written consent was obtained before filling the survey and participants were informed that their participation in the study was entirely voluntary. They were also assured about the confidentiality of the information provided and told that they could withdraw from the study at any time or opt not to answer any of the questions in the survey.

\section{- Consent for publication}

Not applicable

\section{- Availability of data and material}

The datasets generated and analyzed during the current research are not publicly available, but they are available from the corresponding author on reasonable request.

\section{- Competing interests}

The authors declare that they have no competing interests.

\section{- Funding}

This research received no funding.

\section{- Authors' contributions}

Conceptualization: S.H. and A.B.

Methodology: S.H., A.B., K.A. and F.Q.

Software: S.H.

Validation: S.H. and M.A.

Formal analysis: S.H.

Writing-original draft preparation: A.B., K.A., M.A., Y.A. and F.Q.

Writing-review and editing: K.A. M.A, Y.A. and F.Q.

All authors have read and agreed to the published version of the manuscript.

\section{- Acknowledgments}


The authors would like to acknowledge and convey gratitude to Mr. Intisar Siddiquie for his valuable statistical contribution in analyzing the data.

\section{Abbreviations}

- Severe acute respiratory syndrome coronavirus 2 (SARS-CoV-2)

- Coronavirus disease 2019 (COVID-19).

- World Health Organization (WHO)

- Personal Protective Equipment (PPE)

\section{References}

1. Zhu N, et al. A novel coronavirus from patients with pneumonia in China, 2019. N Engl J Med 2020;382:727-733.

2. She J, et al. 2019 novel coronavirus of pneumonia in Wuhan, China: emerging attack and management strategies. Clin Transl Med 2020;9:19.

3. Cascella M, et al. Features, evaluation and treatment coronavirus (COVID-19). In StatPearls. 2020: Treasure Island (FL).

4. Zhou P, et al. A pneumonia outbreak associated with a new coronavirus of probable bat origin. Nature, 2020;579:270-273.

5. Huang C, et al. Clinical features of patients infected with 2019 novel coronavirus in Wuhan, China. Lancet 2020;395:497-506.

6. Wang D, et al. Clinical characteristics of 138 hospitalized patients with 2019 novel coronavirus-infected pneumonia in Wuhan, China. JAMA 2020.

7. Lu CW, Liu XF, Jia ZF. 2019-nCoV transmission through the ocular surface must not be ignored. Lancet 2020;395:e39.

8. Kampf G, et al. Persistence of coronaviruses on inanimate surfaces and their inactivation with biocidal agents. J Hosp Infect 2020;104:246-251.

9. Chen J. Pathogenicity and transmissibility of 2019-nCoV-A quick overview and comparison with other emerging viruses. Microbes Infect 2020;22:69-71.

10. Cleveland JL, et al. Transmission of blood-borne pathogens in US dental health care settings: 2016 update. J Am Dent Assoc 2016;147:729-38.

11. Harrel SK, Molinari J. Aerosols and splatter in dentistry: a brief review of the literature and infection control implications. J Am Dent Assoc 2004;135:429-37.

12. Liu L, et al. Epithelial cells lining salivary gland ducts are early target cells of severe acute respiratory syndrome coronavirus infection in the upper respiratory tracts of rhesus macaques. J Virol 2011;85:4025-30.

13. Saudi Ministry of Health (MOH) COVID-19 Awareness Guidelines. Available from: https://www.moh.gov.sa/awarenessplateform/VariousTopics/Documents/PreventCOVID19-Eng.pdf. Accessed on April 20, 2020.

14. WHO Coronavirus disease 2019 (COVID-19) Situation Report - 88. Available from: https://www.who.int/docs/defaultsource/coronaviruse/situation-reports/20200417-sitrep-88-covid-191b6cccd94f8b4f219377bff55719a6ed.pdf? sfvrsn=ebe78315_6. Accessed on April 20, 2020.

15. Gaffar BO, et al. Knowledge and practices of dentists regarding MERS-CoV. A cross-sectional survey in Saudi Arabia. Saudi Med J 2019;40:714-720.

16. Otter JA, et al. Transmission of SARS and MERS coronaviruses and influenza virus in healthcare settings: the possible role of dry surface contamination. J Hosp Infect 2016;92:235-50.

17. Peng X, et al. Transmission routes of 2019-nCoV and controls in dental practice. Int J Oral Sci 2020;12:9.

18. Saudi Census. Available from: https://saudicensus.sa/. Accessed on April 20, 2020.

\section{Tables}




\begin{tabular}{|c|c|}
\hline Variable & $\mathrm{n}(\%)$ \\
\hline \multicolumn{2}{|l|}{ Age (years) } \\
\hline 20 to $<30$ & $507(50 \%)$ \\
\hline 30 to $<40$ & $301(29.7 \%)$ \\
\hline 40 to $<50$ & $134(13.2 \%)$ \\
\hline 50 or above & $71(7 \%)$ \\
\hline \multicolumn{2}{|l|}{ Profession } \\
\hline Dental student & $197(19.5 \%)$ \\
\hline Dental intern & $76(7.5 \%)$ \\
\hline Dental assistant & $156(15.4 \%)$ \\
\hline Dental hygienist & $37(3.7 \%)$ \\
\hline Postgraduate resident & $92(9.1 \%)$ \\
\hline General dentist & $181(17.9 \%)$ \\
\hline Specialist/consultant & $271(26.8 \%)$ \\
\hline \multicolumn{2}{|l|}{ Region } \\
\hline Northern Region & $46(4.5 \%)$ \\
\hline Southern Region & $107(10.6 \%)$ \\
\hline Western Region & $226(22.3 \%)$ \\
\hline Eastern Region & $367(36.2 \%)$ \\
\hline Central Region & $267(26.4 \%)$ \\
\hline \multicolumn{2}{|l|}{ Work sector } \\
\hline Academia & $336(33.4 \%)$ \\
\hline Government & $452(44.9 \%)$ \\
\hline Private & $219(21.7 \%)$ \\
\hline \multicolumn{2}{|l|}{ Work setting } \\
\hline Hospital & $641(63.4 \%)$ \\
\hline Nonhospital & $370(36.6 \%)$ \\
\hline
\end{tabular}




\begin{tabular}{|c|c|c|c|c|c|c|c|c|c|}
\hline \multirow[t]{2}{*}{ Variable } & \multicolumn{3}{|c|}{ Work setting } & \multirow[t]{2}{*}{$\mathbf{p}$} & \multicolumn{4}{|c|}{ Work sector } & \multirow[t]{2}{*}{ p } \\
\hline & Hospital & Nonhospital & Total & & Academia & Government & Private & Total & \\
\hline \multicolumn{10}{|l|}{$\begin{array}{l}\text { Q1: Incubation period of } \\
\text { SARS-CoV-2? }\end{array}$} \\
\hline $1-7$ dave & & & 27 & 0.587 & & & & 27 & 0.648 \\
\hline 14 dom & 18 & 9 & 924 & & 8 & 10 & 9 & 920 & \\
\hline $1-14$ days & 582 & 342 & 51 & & 309 & 418 & 193 & 51 & \\
\hline 1-21 days & 34 & 17 & 7 & & 16 & 21 & 14 & 7 & \\
\hline Do not know & 6 & 1 & & & 3 & 2 & 2 & & \\
\hline Total & 640 & 369 & & & 336 & 451 & 218 & & \\
\hline \multicolumn{10}{|c|}{$\begin{array}{l}\text { Q2: Survival of SARS-CoV-2 } \\
\text { outside the host? }\end{array}$} \\
\hline \multirow{2}{*}{ Couple of hours } & & & 496 & & & & & 496 & \\
\hline & 323 & 173 & 360 & 0.146 & 163 & 222 & 111 & 357 & 0.083 \\
\hline Couple of days & 213 & 147 & 71 & & 133 & 157 & 67 & 71 & \\
\hline Couple of weeks & 46 & 25 & 82 & & 15 & 39 & 17 & 81 & \\
\hline Do not know & 58 & 24 & & & 25 & 32 & 24 & & \\
\hline Total & 640 & 369 & & & 336 & 450 & 219 & & \\
\hline \multicolumn{10}{|c|}{$\begin{array}{l}\text { Q4: Recommended hand- } \\
\text { soap cleaning time to } \\
\text { prevent SARS-CoV-2 } \\
\text { infection? }\end{array}$} \\
\hline Approximately $20 \mathrm{~s}$ & & & 276 & 0.244 & & & & 275 & 0.060 \\
\hline \multirow{2}{*}{ Approximately $40 \mathrm{~s}$} & 163 & 113 & 560 & & 95 & 124 & 56 & 558 & \\
\hline & 359 & 201 & 155 & & 179 & 251 & 128 & 154 & \\
\hline Approximately $60 \mathrm{~s}$ & 106 & 49 & 17 & & 48 & 73 & 33 & 17 & \\
\hline Do not know & 10 & 7 & & & 12 & 3 & 2 & & \\
\hline Total & 638 & 370 & & & 334 & 451 & 219 & & \\
\hline \multicolumn{10}{|c|}{$\begin{array}{l}\text { Q4: Willingness to treat a } \\
\text { suspected COVID-19 patient? }\end{array}$} \\
\hline \multicolumn{10}{|l|}{ Yes } \\
\hline \multirow{2}{*}{ No } & 164 & 87 & 251 & 0.489 & 90 & 173 & $4 /$ & 250 & 0.358 \\
\hline & 475 & 280 & 755 & & 244 & 337 & 171 & 752 & \\
\hline Total & 639 & 367 & & & 334 & 450 & 218 & & \\
\hline
\end{tabular}




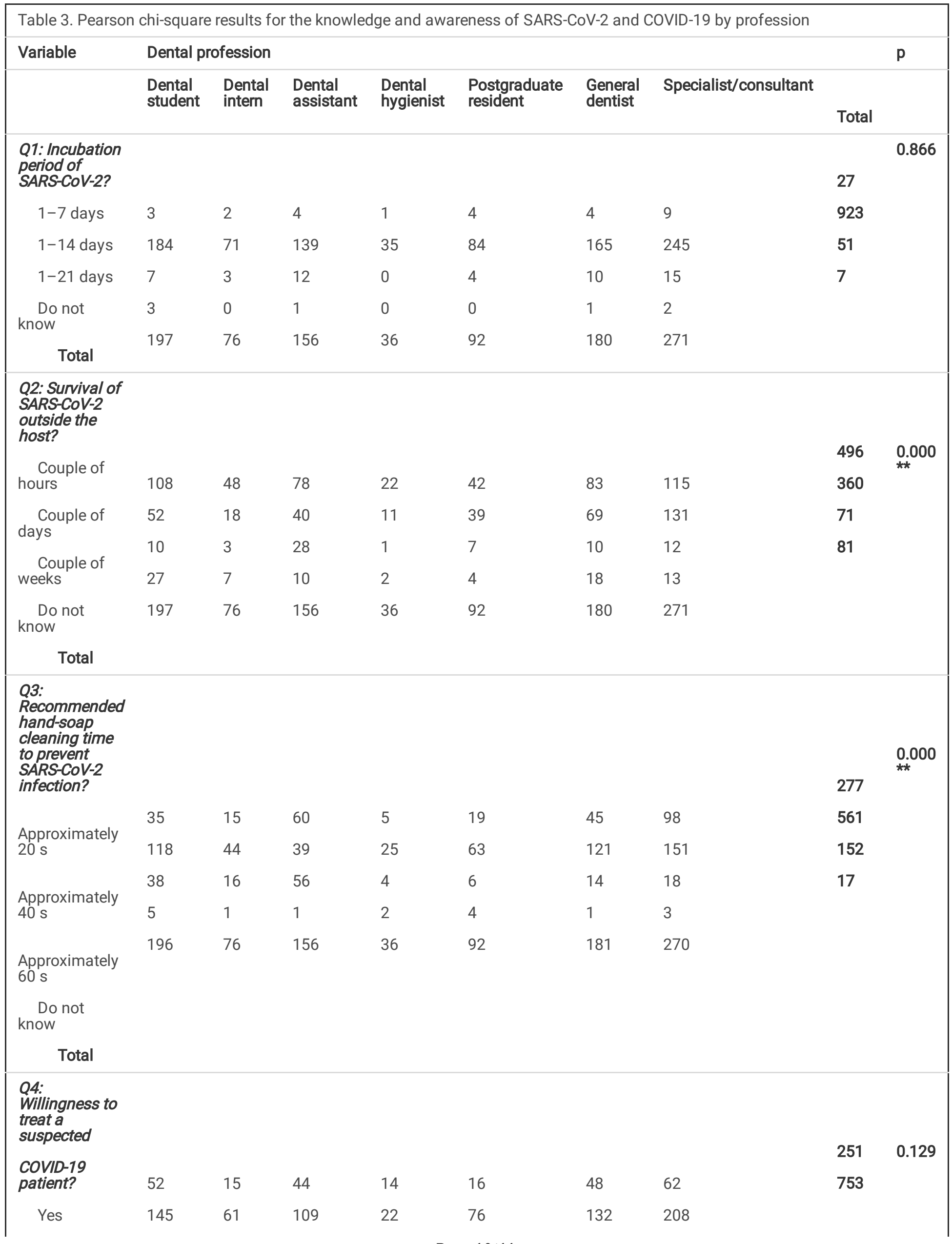


Total

All tests were performed at a significance level of $a=0.05$.

*Significant at $\mathrm{p}<0.05$. **Significant at $\mathrm{p}<0.001$.

Table 4. A summary of the awareness of the route of SARS-CoV-2 transmission, signs and symptoms of COVID-19, and methods of SARS-CoV-2 transmission prevention in dental clinics

\section{Q5: Route of SARS-CoV-2 transmission}

Direct skin-to-skin transmission Frequency Percent

s, or blood)

(2)

312 $30.8 \%$

Direct fluid transmission (saliva, body fluids, or blood)

Droplet spread (sneezing or coughing)

Airborne transmission (air or dust)

Q6: Signs and symptoms of COVID-19

Fever

Headache

Diarrhea

Vomiting

\section{8}

932

305

Frequency

1,000

736

323

187

492

944

940

671

295

32

Skin rash

Q7: Methods of SARS-CoV-2 transmission prevention in dental clinics

Hand-soap cleaning

Frequency

Percent

Hand sanitizers

960

873

973

360

207

620

877

579

592

$54.1 \%$

$92 \%$

$30.1 \%$

Percent

$98.7 \%$

$72.7 \%$

$31.9 \%$

$18.5 \%$

$48.6 \%$

$93.2 \%$

$92.8 \%$

$66.2 \%$

$29.1 \%$

$3.2 \%$

$94.8 \%$

$86.2 \%$

$96.1 \%$

$35.5 \%$

$20.4 \%$

$61.2 \%$

Clinic surface disinfection

Adequate ventilation

Utilization of an isolated clinic
$86.6 \%$

$57.2 \%$

$58.4 \%$ 